\title{
Evaluation De La Durabilité Agro-Écologique Des Aménagements Hydro-Agricoles De La Plaine Inondable Dans Le Tandem Dangbo-Adjohoun Au Sud-Est Du Bénin
}

\author{
Mouritala Abou \\ Département de Géographie et Aménagement du Territoire, \\ DGAT/FASHS/UAC, Cotonou, Bénin \\ Ibouraïma Yabi \\ Euloge Ogouwale \\ Laboratoire Pierre Pagney, Climat, Eau, \\ Ecosystèmes et Développement (LACEEDE)
}

Doi: 10.19044/esj.2018.v14n9p226 URL:http://dx.doi.org/10.19044/esj.2018.v14n9p226

\begin{abstract}
Sustainable agriculture has become a priority in the public policies of countries like Benin, where rural people live mainly from agricultural products. However, agricultural development strategies are geared towards practices that do not meet the objectives of sustainability. This paper focuses on assessing the agro-ecological sustainability of the perimeters developed in the flood plain of Dangbo Commune and Adjohoun. In the Ouémé valley, this plain facilitates the development of a variety of hydro-agricultural developments whose agro-ecological sustainability of production conditions has been analyzed through the IDEA (Farm Durability Indicator) method. Field surveys carried out in eight 8 districts (Késsounou, Houédomey, Gbéko, Zounguè, Gangban, Kodé, Démè and Azowlissè) made it possible, using a questionnaire, to collect the qualitative and quantitative data from 254 operators of the agricultural hydro planning sites. 20 developed perimeters selected among the 66 were selected to evaluate their agro-ecological sustainability. The processing of the collected data is done according to the methods of determining the levels of sustainability. This sustainability, evaluated by thirteen indicators, thus grouping three components of the scale, yielded an average score of 58.72 out of 100 . Overall, the diversity component 23.88 and the organization of space 22,79 achieved high average scores, thus contributing to the agro-ecological sustainability of the developed perimeters. On the other hand, the "agricultural practices" 12,05 constitute a component of which the lines of reflection and improvement are possible through
\end{abstract}


indicators "fertilization, pesticides and protection of the ground resource" for a sustainable hydro-agricultural development.

Keywords: Low valley of the Ouémé (South-Benin), agricultural hydro amenities, agro-ecological long lasting, IDEA method

\section{Résumé}

L'agriculture durable est devenue une priorité des politiques publiques des pays comme le Bénin où les populations rurales vivent essentiellement des produits agricoles. Cependant, les stratégies de développement agricole sont orientées vers des pratiques qui ne répondent pas aux objectifs de la durabilité. La présente recherche a pour but d'évaluer la durabilité agro-écologique des périmètres aménagés dans la plaine inondable de la Commune de Dangbo et d'Adjohoun. Dans la vallée de l'Ouémé, cette plaine facilite le développement d'une diversité d'aménagements hydro-agricoles dont la durabilité agroécologique des conditions de production a été analysée à travers la méthode IDEA (Indicateur de Durabilité des Exploitations Agricoles). Les enquêtes de terrain réalisées dans huit 8 arrondissements (Késsounou, Houédomey, Gbéko, Zounguè, Gangban, Kodé, Démè et Azowlissè) ont permis de recueillir, à l'aide d'un questionnaire, des informations qualitatives et quantitatives auprès de 254 exploitants des sites d'aménagement hydroagricole. Vingt 20 périmètres aménagés choisis parmis les 66 ont été retenus pour évaluer leur durabilité agro-écologique. Le traitement des données collectées est fait en suivant les modalités de détermination des niveaux de durabilité. Cette durabilité, évaluée par treize 13 indicateurs regroupant ainsi trois 3 composantes de l'échelle, a permis d'obtenir un score moyen de 58,72 sur 100. Globalement, la composante «diversité » 23,88 et « organisation de l'espace » 22,79 ont obtenu des scores moyens élevés contribuant ainsi à la durabilité agro-écologique des périmètres aménagés. Par contre, les «pratiques agricoles »12,05 constituent une composante dont les pistes de réflexion et d'amélioration sont envisageables à travers des indicateurs «fertilisation, pesticides et protection de la ressource sol » pour un aménagement hydro-agricole durable.

Mots-clés : Basse vallée de l'Ouémé (Sud-Bénin), aménagements hydroagricoles, durabilité agro-écologique, méthode IDEA

\section{Introduction}

L'agriculture constitue une ressource territoriale historique majeure des pays du tiers monde qui contribue au bien-être humain en produisant,entre autres, de la nourriture, des matières de base et de l'emploi (Terrier, 2010). Elle participe au développement économique de ces pays et reste, en général, 
l'activité principale des populations. C'est une potentialité spécifique des territoires ruraux fortement agricoles encore (Pierre et al., 2008). En Afrique de l'Ouest, l'agriculture a un poids économique particulier puisque dans la zone de l'Union Economique et Monétaire Ouest Africaine (UEMOA), elle contribue pour $30 \%$ au Produit Intérieur Brut (PIB) et emploie plus de $50 \%$ de la population active (Renard et al., 2004). Le Bénin dispose de 4.814.000 ha de terres réeellement cultivables et près de 6.700 ha de plans d'eau dont 2.000 ha de fleuves : environs 1.900 ha de lacs et un système lagunaire de plus de 2.800 ha (MAEP, 2009). Ces atouts naturels facilitent le développement d'une agriculture durable. Les Communes de Dangbo et d'Adjohoun disposent également d'une importante quantité de ressources en eaux superficielles, surtout dans la plaine inondable du fleuve Ouémé, qui permettent le développement des aménagements hydro-agricoles (Vissin, 2013). Ces Communes, de part leurs caractéristiques physiques et humaines, valorisent la plaine à travers les types d'aménagements hydro-agricoles: irrigation, drainage et la construction des barrages hydro-agricoles (Codjo, 2014). Il importe que l'activité agricole assure, aussi bien, une fonction économique de production de biens et de services, soutenant directement ou indirectement la création d'emplois ruraux, une fonction sociale d'occupation du territoire, d'animation du monde rural et de transmission d'un patrimoine culturel spécifique de même qu'une fonction écologique de protection de l'environnement et d'entretien de l'espace rural (Landais, 1998 cité par Ligan, 2015). Pourtant, il est aujourd'hui démontré par Steinfed et al. (2006) que de nombreuses pratiques agricoles actuelles ont des conséquences néfastes sur notre environnement: dégradation des sols, pollutions des eaux, appauvrissement de la biodiversité, changement climatique. Ces comportements ne sont pas de nature à garantir la durabilité des aménagements hydro-agricoles. Or, le développement des exploitations agricoles plus durable est donc aujourd'hui une priorité (Lamothe, 2007). Il est impérieux de penser aux marges de progrès en évitant le pire afin de garantir la durabilité des aménagements hydro-agricoles.

\section{Matériel et méthodes}

\section{Caractérisation du mileu de recherche}

Situées dans le département de l'Ouémé entre $6^{\circ} 36^{\prime}$ et $6^{\circ} 43^{\prime}$ de latitude nord et entre $2^{\circ} 21^{\prime}$ et $2^{\circ} 35^{\prime}$ de longitude est, les Communes de Dangbo et d'Adjohoun sont limitées au nord par la Commune de Bonou, au sud par la Commune des Aguégués, à l'est par la Commune de Sakété et à l'ouest par la Commune de Zè (Figure 1). Elles couvrent une superficie de 457 $\mathrm{km}^{2}$ (INSAE/RGPH, 2002). 


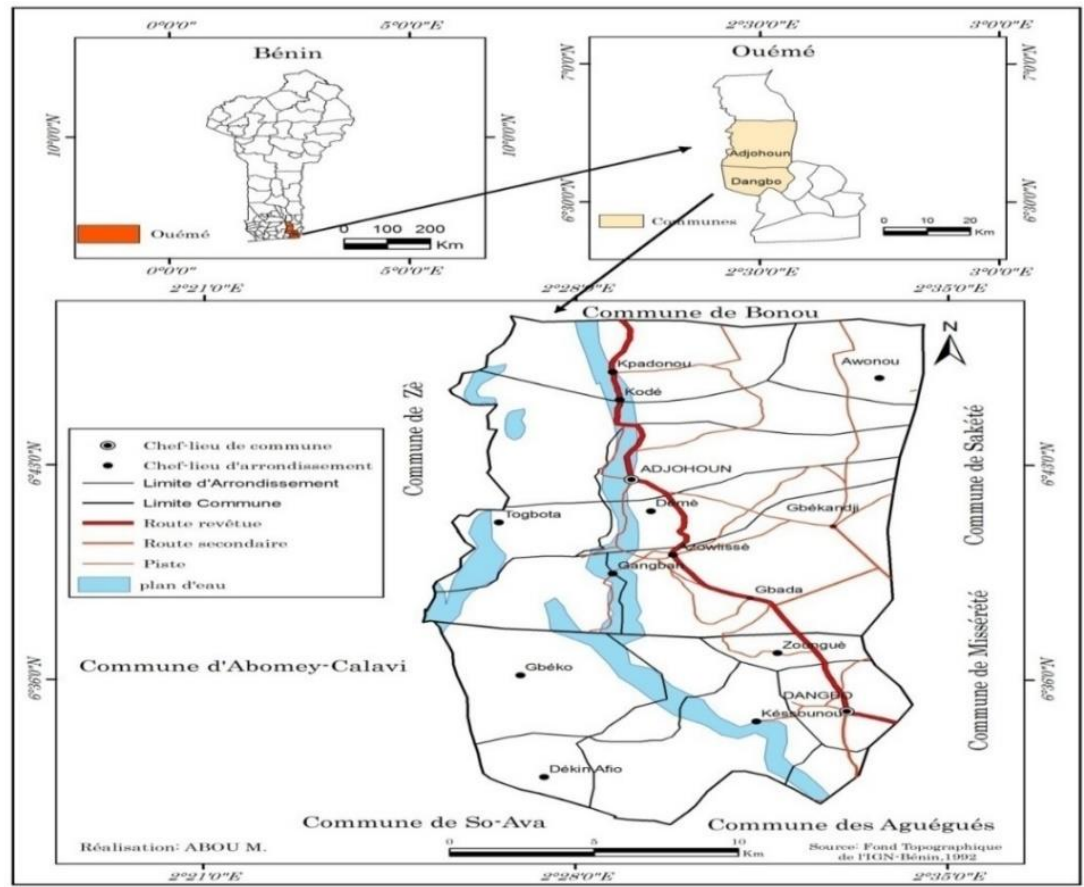

Figure 1. Situation géographique et subdivisions administratives de la zone d'étude

Le relief est composé de deux unités géomorphologiques : un plateau d'altitude comprise entre 20 et $200 \mathrm{~m}$ présente des ondulations moyennes fortes et une plaine inondable ne dépassant pas $10 \mathrm{~m}$ d'altitude d'axe nord-sud qui, dans la topo séquence est-ouest, jouxte le plateau. Elle s'étend de part et d'autre du fleuve Ouémé qui l'inonde annuellement entre les mois de juillet et novembre. Sur la plaine inondable, il existe des sols alluviaux et colluviaux dans le lit du cours d'eau et des vertisols dans les marécages. Les conditions hydriques des sols de la plaine de Dangbo et d'Adjohoun sont très favorables aux activités de production agricole (Boko, 1988).

L'hydrographie quant à elle est composée du fleuve Ouémé qui entre ensuite dans les formations sédimentaires du bas-Bénin et coule dans les alluvions récentes qui le bordent de part et d'autre. Sa pente est alors très faible et les eaux se dispersent par de nombreux bras inondant de vastes plaines, donnant lieu au delta de l'Ouémé et communiquant en plusieurs endroits avec le lac Nokoué et la lagune de Porto-Novo (Lalèyè, 1995). Le fleuve Ouémé, d'une superficie de $4980 \mathrm{~km}^{2}$, dispose de 60000 ha de terre irrigable. Le milieu de recherche dispose de 5547350 ha environ de terre cultivable, 7466 ha de terre aménageable et 2990 ha de terre aménagée par le Projet d'Appui à la Diversification Agricole (PADA) et le Programme d'Appui aux Infrastructures Agricoles dans la Vallée de l'Ouémé (PAI-VO) ainsi que les producteurs, soit $40 \%$ des terres aménageables (Secteur Communal pour le Développement Agricole/Adjohoun, 2016). 
En ce qui concerne le climat, il est caractérisé par un régime à quatre saisons. Il y a une grande saison des pluies de mars à juin (4 mois), une courte saison sèche de juillet à août (2mois), une courte saison de pluies commençant en septembre et terminant t en octobre ( 2 mois) et une longue saison sèche du mois de novembre à février (4 mois). Ce climat est proche du climat subéquatorial par l'abondance des précipitations. Les précipitations moyennes annuelles varient entre $1200 \mathrm{~mm}$ et $1600 \mathrm{~mm}$ (Adam \& Boko, 1993). Elles contribuent parfois à l'inondation du fleuve Ouémé facilitant ainsi le développement des aménagements hydro-agricoles sur la plaine d'inondation du milieu de recherche.

\section{Méthodes de collecte et de traitement des données}

Il s'agit des informations qualitatives et quantitatives recueillies auprès des exploitants agricoles sur les composantes (diversité, organisation de l'espace et pratiques agricoles) à travers les différents indicateurs de l'échelle de la durabilité agro-écologique des aménagements hydro-agricoles.

Les enquêtes de terrain ont été réalisées dans huit (08) arrondissements (Késsounou, Houédomey, Gbéko, Zounguè, Gangban, Kodé, Démè et Azowlissè) sur les quinze (15) que la zone d'étude comprend. L'échantillonnage raisonné des arrondissements a été effectué en suivant les critères de proximité du fleuve Ouémé qui facilite les activités hydro-agricoles sur la plaine d'inondation. La taille de l'échantillon a été déterminée grâce au protocole de Schwartz (1995) par la formule : $\mathrm{n}=\mathbf{Z} \boldsymbol{\alpha}^{\mathbf{2}} \mathbf{x} \mathbf{~ p q} / \mathbf{i}^{\mathbf{2}}$ avec : $\mathbf{Z} \boldsymbol{\alpha}=1,96$ : écart réduit correspondant à un risque $\alpha$ de $5 \% ; \mathbf{p}=\mathrm{n} / \mathrm{N}$ étant $\mathrm{p}$ la proportion des ménages qui développent les aménagements hydro-agricoles par rapport aux ménages totaux dans les Communes d'étude $\mathbf{i}=$ précision désirée égale à $6 \% ; \mathbf{q}=1-\mathrm{p}, \mathbf{n}=(1,96)^{2} \times \mathrm{p}(1-\mathrm{p}) / 0,05^{2}$. Ainsi, le choix des périmètres aménagés qui ont servi à l'évaluation de la durabilité agro-écologique, est fait de façon raisonnée dans les exploitations agricoles parcourues dans la zone d'étude (tableau I).

Tableau I. Nombre de périmètres aménagés retenus dans les arrondissements d'étude

\begin{tabular}{ccccc}
\hline Arrondissements & $\begin{array}{c}\text { Périmètres } \\
\text { total } \\
\text { aménagés }\end{array}$ & $\begin{array}{c}\text { Périmètres } \\
\text { aménagés } \\
\text { retenus }\end{array}$ & $\begin{array}{c}\text { Nombre de } \\
\text { ménages } \\
\text { agricoles }\end{array}$ & $\begin{array}{c}\text { Nombre de ménages } \\
\text { agricoles/aménagement } \\
\text { retenus }\end{array}$ \\
\hline Gangban & 09 & 04 & 31664 & 38 \\
\hline Kodé & 05 & 02 & 1241 & 15 \\
Démè & 03 & 03 & 520 & 06 \\
Azowlissè & 08 & 03 & 4701 & 56 \\
\hline Gbéko & 09 & 02 & 2777 & 33 \\
Houédomey & 12 & 03 & 3653 & 44 \\
Késsounou & 15 & 03 & 2870 & 28 \\
Zounguè & 05 & 00 & 2382 & $\mathbf{2 5 4}$ \\
\hline 08 & 66 & $\mathbf{2 0}$ & $\mathbf{2 1 3 0 8}$ & \\
\hline
\end{tabular}

Source : INSAE, RGPH4 et SCDA-Dangbo/Adjohoun, 2017 
Au total, 20 périmètres aménagés ont été retenus pour l'évaluation de la durabilité agro-écologique des aménagements hydro-agricoles sur les 254 exploitants interviewés.

La grille d'observation a permis d'observer les pratiques agricoles, l'organisation de l'espace et la diversité des productions sur les périmètres aménagés.

\section{Méthodes de traitement des informations Processus d'utilisation de la méthode IDEA}

La méthode IDEA est un outil qui permet d'évaluer la durabilité des systèmes d'exploitation agricole. Cette évaluation se base sur la durabilité agro-écologique, la durabilité socio-territoriale et la durabilité économique de l'exploitation agricole aménagée. Chaque échelle est subdivisée en 3 ou 4 composantes comportant 41 indicateurs de durabilité. La méthode retient des notes maximales 14 ou minimales 0 pour chaque indicateur, de façon à plafonner le nombre total d'unités de durabilité se rapportant à l'un des domaines étudiés. La notation des trois échelles de la durabilité varie sur une gamme de 0 à 100 points (Vilain et al., 2000). Le score d'une exploitation pour chacune des trois échelles de durabilité est le nombre cumulé d'unités élémentaires de durabilité obtenues dans les divers domaines tenus en compte dans cette échelle. La détermination du score de l'indicateur «Diversité des cultures annuelles ou temporaires » est obtenu en suivant la procédure comme l'indique le tableau II.

Tableau II. Données et modalités de détermination des niveaux de durabilité agro-

\begin{tabular}{|c|c|c|c|c|}
\hline Indicateur & Données & $\begin{array}{c}\text { Modalités de détermination du } \\
\text { niveau de durabilité }\end{array}$ & Score & $\begin{array}{l}\text { Valeur } \\
\text { plafond }\end{array}$ \\
\hline $\begin{array}{c}\text { Diversité } \\
\text { des cultures } \\
\text { annuelles ou } \\
\text { temporaires }\end{array}$ & $\begin{array}{l}\text { Espèces cultivées } \\
\text { variétés de chaque } \\
\text { espèce } \\
\text { superficies par } \\
\text { espèce cultivée } \\
\text { présence de } \\
\text { prairies :(superficie } \\
\text { et âges) }\end{array}$ & $\begin{array}{c}\text { Par espèce cultivée } \\
\text { si il y a plus de six variétés au total } \\
\text { en cas de présence de légumineuse } \\
\text { dans l'assolement de } 5 \text { à } 10 \% \\
\text { en cas de présence de légumineuse } \\
\text { dans l'assolement de } 10 \text { à } 15 \% \\
\text { en cas de présence de légumineuse } \\
\text { dans l'assolement plus de } 15 \%\end{array}$ & $\begin{array}{l}2 \\
2 \\
1 \\
2\end{array}$ & 14 \\
\hline
\end{tabular}

Source : Conçu sur la base des modalités de détermination de la durabilité proposées par la méthode IDEA de Vilain (2008)

Le tableau II présente les données et modalités de détermination du niveau de durabilité ainsi que le score et la valeur plafond de l'indicateur. Ainsi, l'évaluation du score de durabilité d'un périmètre aménagé est obtenue de la manière suivante :

- $\quad$ Nombre d'espèces cultivées : 4 ; score de durabilité $=8$ 
- $\quad$ Nombre de variétés cultivées : 7 ; score de durabilité = 2

- $\quad$ Proportion de légumineuse dans l'assolement : 1; score de durabilité $=1$

Score total de durabilité pour l'indicateur $=8+2+1=11$

Ainsi, plus la note est élevée, plus l'exploitation est considérée comme durable pour l'échelle examinée. Les notes maximales traduisent le poids accordé à chaque indicateur au sein du domaine auquel il se rapporte et ,par conséquent, le poids accordé à chaque domaine dans les trois échelles de durabilité concernées. Ainsi, les indicateurs fondamentaux ayant plus de poids que les indicateurs spécifiques sont classés entre eux. Toutefois, il n'est pas rare d'attribuer à certains indicateurs causant des préjudices sur la durabilité des notes négatives causant. Au sein d'une échelle donnée, les poids des divers domaines sont soit identiques (échelles agro-écologiques et échelle socioterritoriale) soit légèrement différents (échelle économique). Dans le cadre de cette étude, il sera question de l'évaluation de la durabilité agro-écologique des aménagements hydro-agricoles de la plaine inondable des Communes de Dangbo et d'Adjohoun.

\section{Traitement et analyse des informations}

Les informations collectées sur le terrain ont été traitées en suivant les modalités de détermination du niveau de durabilité proposées par la méthode IDEA. De ce fait, la contribution des indicateurs et composantes dans la durabilité agro-écologique a été analysée.

\section{Résultats}

Les indicateurs de durabilité de chaque composante ont déterminé les conditions actuelles de production dans les périmètres aménagés de la zone d'étude (Tableau III et Figure 2).

Tableau III. Scores moyens de l'échelle de la durabilité agro-écologique

\begin{tabular}{|c|c|c|c|}
\hline Composantes & Indicateurs & $\begin{array}{c}\text { Scores } \\
\text { moyens }\end{array}$ & $\begin{array}{c}\text { Valeurs } \\
\text { maximales }\end{array}$ \\
\hline \multirow{4}{*}{ Diversité } & $\begin{array}{c}\text { Diversité des cultures annuelles et } \\
\text { temporaires }\end{array}$ & 11,2 & 14 \\
\cline { 2 - 4 } & Diversité des cultures pérennes & 5,58 & 14 \\
\cline { 2 - 4 } & Diversité animale & 1,16 & 14 \\
\cline { 2 - 4 } & $\begin{array}{c}\text { Valorisation et conservation du } \\
\text { patrimoine génétique }\end{array}$ & 5,94 & 6 \\
\hline \multirow{3}{*}{$\begin{array}{c}\text { Organisation de } \\
\text { l'espace }\end{array}$} & Assolement & 7,8 & 10 \\
\cline { 2 - 4 } & Dimension des parcelles & 4,8 & 6 \\
\cline { 2 - 4 } & Gestion des matières organiques & 1,6 & 5 \\
\cline { 2 - 4 } & Zone de régulation écologique & 7,6 & 10 \\
\cline { 2 - 4 } & Gestion des surfaces fourragères & 0,99 & 3 \\
\hline
\end{tabular}




\begin{tabular}{|c|c|c|c|}
\hline \multirow{3}{*}{$\begin{array}{c}\text { Pratiques } \\
\text { agricoles }\end{array}$} & Fertilisation & 1,6 & 8 \\
\cline { 2 - 4 } & Pesticides & 2,8 & 10 \\
\cline { 2 - 4 } & Protection de la ressource sol & 1,85 & 5 \\
\cline { 2 - 4 } & Gestion des ressources en eau & 5,8 & 10 \\
\cline { 2 - 4 } & Total général & 58,72 & 100 \\
\hline
\end{tabular}

Source :Enquêtes de terrain, janvier 2017

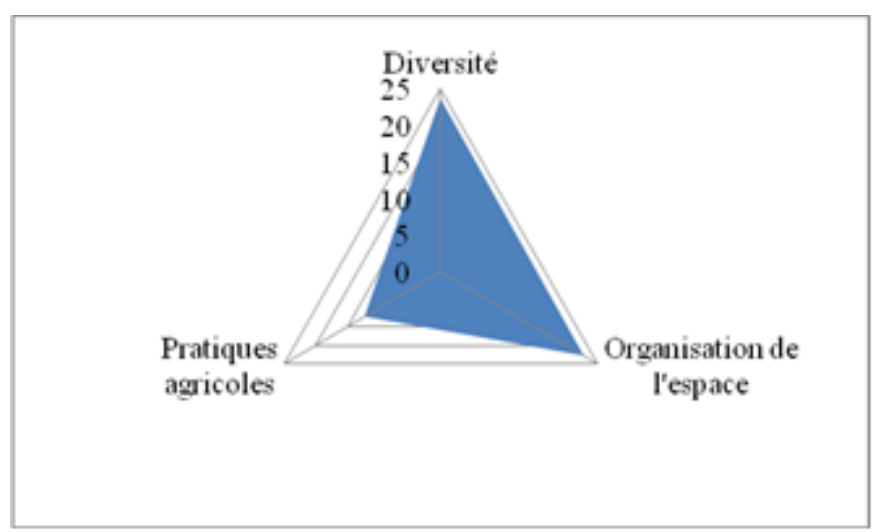

Figure 2. Répresentation graphique des composantes de la durabilité des périmètres aménagés

Le Tableau III présente le total des scores moyens (58,72 sur 100) des 20 périmètres aménagés considérés dans l'échantillonnage. $\mathrm{Vu}$ ces valeurs obtenues, il ressort que seuls les indicateurs de durabilité « diversité $(23,88)$ » et « organisation de l'espace $(22,79) »$ ont participé largement à la durabilité agro-écologique tandis que les «pratiques agricoles $(12,05) »$ constituent les pistes de réflexion et d'amélioration pour des périmètres aménagés durables dans la zone d'étude.

\section{Composante « diversité domestique »}

L'échelle de la durabilité agro-écologique analyse les systèmes de production au sein des aménagements hydro-agricoles à travers une valorisation du milieu et une stabilité écologique. Il ressort de l'analyse du tableau II que dans la composante diversité, l'indicateur des cultures annuelles ou temporaires a obtenu une valeur plus élevée $(11,2)$. Ce score témoigne de la biodiversité végétale dont regorgent les périmètres aménagés de la plaine inondable. En effet, il s'agit des céréales (Oryza sp, Zea mays), des racines et tubercules (Manihot esculenta, Ipomoea batatas), des légumineuses (Vigna unguculata, et Hrachis hypogea) et les cultures maraîchères (Abelmoschus esculentus, Lycopersicon esculentum, Capcicum spp., Vernonia amydilan), etc. Ce qui lui a valu 2 points par espèce et 2 points si plus de 6 variétés. La présence de ses espèces contribue à la fertilité du sol à long terme en le protégeant contre l'érosion. D'où le score $(5,58)$ obtenu par l'indicateur des 
cultures pérennes qui peut s'expliquer par la présence d'arboriculture isolement répandu sur la plaine et des forêts galeries le long des berges. Les prairies permanentes et temporaires observées sont moins de $10 \%$ de la surface totale utilisée. Aussi, l'inexistence de l'agroforesterie et les cultures ou prairies sous verger ne permettent-ils pas de renforcer la durabilité agronomique et environnementale des périmètres aménagés. Concernant le faible score $(1,16)$ de la diversité animale, sur la plaine, les animaux sauvages sont quasiment inexistants. Ils sont limités aux rongeurs et reptiles. 6 sur les 20 périmètres ont chacun obtenu une note de 2 . Enfin, la valorisation et la conservation du patrimoine génétique vise à conserver les espèces cultivées localement. De nos jours, ses espèces cultivées existent mais elles sont un peu rares. Il s'agit du maïs Zea mays de couleur rouge et de niébé Vigna unguculata appelés en langue locale respectivement «agbokoun » et «wakoun; sôwétin ».

\section{Composante «Organisation de l'espace »}

Dans cette composante, les indicateurs gestion des surfaces fourragères, gestion des matières organiques et dimension des parcelles indiquent des scores faibles. Par contre, ces scores sont élevés dans les indicateurs zones de régulation écologique et assolement. Sur les périmètres aménagés, la surface de la principale culture annuelle est inférieure à $35 \%$ de la surface assolable. Pour $85 \%$ des enquêtés, l'assolement qui se pratique sur les cultures maraîchères (gombo, piment, légumes et tomate) est d'une importance capitale dans la mesure où les bénéfices issus de la vente des produits permettent de répondre aux besoins vitaux en période de soudure (période de crue), ce qui lui confère un score de $(7,8)$ sur 10 points. L'objectif de durabilité de l'assolement est la protection du sol et des êtres vivants et la diversification des cultures. Cet assolement, pratiqué par les exploitants agricoles, est d'un caractère simplifié. Toutefois, la méthode IDEA alerte les agriculteurs sur les risques liés aux assolements simplifiés qui généralement altèrent biologiquement les sols. Le fleuve Ouémé, avec une longueur de 4 $980 \mathrm{~km}^{2}$ et disposant de 60000 ha de terre irrigable (Lègba, 2006), témoigne de la présence de point d'eau et de zone humide à Dangbo et Adjohoun. Aucun périmètre ne dispose des parcelles en jachère de plus de cinq ans. Ce qui peut expliquer le score élevé $(7,6)$ de l'indicateur zone de régulation écologique. Par contre, l'absence d' animaux sauvages et domestiques sur les périmètres aménagés témoigne de la mauvaise gestion des surfaces fourragères $(0,99)$. De même, les matières organiques sont essentiellement constituées des débris végétaux (tiges sèches de maïs laissées après la récolte sur la parcelle et les herbes sèches). Cette technique, pratiquée par $20 \%$ des enquêtés pour les cultures de gombo, de maïs et de piment est observée sur moins de $10 \%$ de la surface agricole utilisée. Le score moyen $(1,6)$ de l'indicateur reflète le faible 
apport annuel des matières organiques. Or, elles sont importantes dans l'entretien et la conservation des sols. Selon les résultats terrain, pour $35 \%$ des périmètres de l'échantillon considéré, la dimension de la parcelle prédominante est supérieure à 6 ha alors que la dimension 6 ha représente le premier intervalle de l'échelle proposée par Vilain (2000) pour cet indicateur. Toutefois, il n'est pas rare d'observer des dimensions inférieures ou égales à 8 ha, d'où le score $(4,8)$ sur 6 points.

\section{Composante «Pratiques agricoles »}

Les indicateurs de cette composante concernent la protection de la ressource sol, la fertilisation, l'utilisation des pesticides et la gestion des ressources en eau (l'irrigation). La fertilisation de la plaine est assurée annuellement par les résidus d'engrais utilisés dans les champs de coton au nord Bénin drainés par le fleuve Ouémé. Pourtant, $75 \%$ des enquêtés utilisent l'urée et le NPK pour plus de rendement à la récolte. Ainsi, près de $100 \mathrm{~kg}$ d'azote sont annuellement utilisés par hectare. L'indicateur fertilisation de score $(1,6)$ contribue à l'appauvrissement des sols. Selon les résultats de terrain, $95 \%$ des enquêtés utilisent les pesticides (herbicides totaux et sélectifs et les insecticides). Les travaux champêtres étant manuels, pour avoir plus de superficies à emblaver sur la plaine inondable, les herbicides totaux (Forge Up ; Sharp ; Herbeextra et Glycel) en provenance du Nigéria, du Ghana et de la Chine permettent aux exploitants d'assécher les herbes à l'aide des pulvérisateurs sans aucune disposition sanitaire. De plus, les insecticides (Clofos de 20\% Ec ; Lama Finer 2,5 Ec ; Termicot 20\% Ec, etc.) mettent les cultures à l'abri des insectes et ravageurs (planche 1). Le dosage parhectare des produits se fait sans aucun respect des normes recommandées par les Secteurs Communaux pour le Développement Agricole de la zone d'étude. Pour les traitements, $90 \%$ épandent les produits par le pulvérisateur et $10 \%$ à travers le bol. Or, prohibés pour le traitement phytosanitaire en maraîchage, ces produits toxiques sont chargés de risques pour les composantes environnementales aussi bien que pour la santé humaine. 


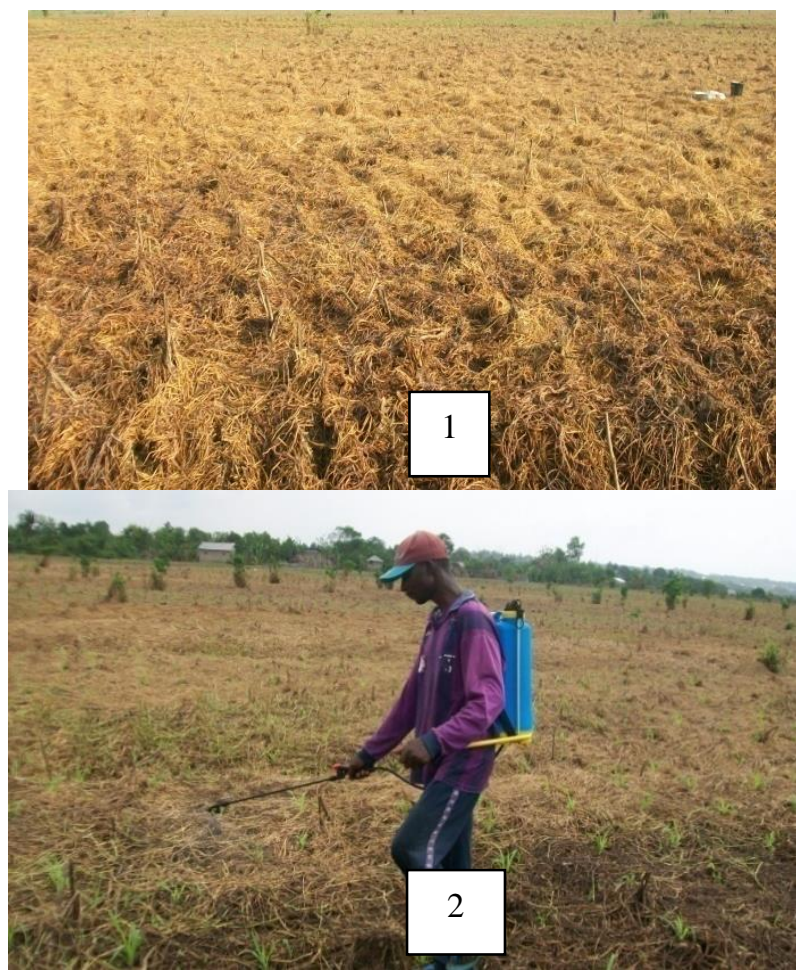

Planche 1. Champs asséché par l'herbicide total (1) et pulvérisation de l'insecticide sur le maïs à Gangban-centre (2)

Prise de vues : Abou, janvier 2017

Cet indicateur varie de 0 à 10 points avec une moyenne de 2,8 . Ce faible score confère une forte pression polluante (comprise entre 4 et 6) des pesticides sur les périmètres aménagés. Ces exploitants agricoles ne font aucune lutte biologique sur la surface utilisée. Une pratique qui ne garantie pas à court et à long terme la protection de la ressource sol. Par ailleurs, l'indicateur gestion des ressources en eau contribue à la durabilité avec un score de 5,8 sur 10 points. En effet, $97 \%$ des enquêtés pratiquent l'aménagement hydro-agricole par irrigation dans les casiers rizicoles et l'aménagement hydro-agricole par drainage aux alentours des cultures irriguées.

Par ailleurs, les pistes de réflexion pour une amélioration impliquent la remise en cause de certaines pratiques agricoles.

\section{Pistes de réflexion pour des aménagements hydro-agricoles durables}

Globalement, les composantes diversité et organisation de l'espace constituent les scores forts de la durabilité agro-écologique des périmètres aménagés. Ces résultats (figure 2) sont la conséquence d'une diversité de cultures annuelles et temporaires, diversité de cultures pérennes, d'une large surface de zones de régulations écologiques et de la répartition des cultures 
sur les mêmes parcelles. Par contre, ces résultats présentent les scores faibles de la composante pratiques agricoles dont les pistes d'amélioration à moyen terme, voire à court terme, portent sur les indicateurs fertilisation, pesticides et protection de la ressource sol.

En effet, le but de la méthode IDEA est de produire sans altérer l'environnement, laquelle est une manière d'aller vers une valorisation de la matière organique produite. Ainsi, les exploitants agricoles doivent privilégier la fertilisation organique au détriment de la fertilisation minérale dans la mesure où la première permette, maintenant et dans l'avenir, d'avoir des sols moins pollués, fertiles et une bonne santé des exploitants agricoles. En un mot, l'utilisation de la fertilisation organique serait une conséquence de la réduction d'azote et une diminution liée à l'achat des engrais chimiques.

Une agriculture écologiquement saine doit chercher à limiter l'utilisation des pesticides qui constituent une menace pour la santé humaine et pour les écosystèmes (Vilain, 2003). De plus, la méthode IDEA condamne l'utilisation abusive des pesticides sur les périmètres aménagés. De ce fait, les pistes d'amélioration de l'utilisation de ces produits sont l'interdiction des pesticides moins chers à qualité douteuse venant d'horizons diverses (Nigéria, Ghana et Togo) sur les marchés locaux de la zone d'étude et la disponibilité à bas prix des produits moins toxiques dans les structures déconcentrées d'Etat (SCDA) de Dangbo et d'Adjohoun. Ensuite, ces structures doivent périodiquement former les exploitants agricoles des périmètres aménagés sur le dosage homologué par hectare et les dispositions sanitaires recommandées dès leur contact avec les produits chimiques. En pratique, lors de l'homologation des pesticides, il est calculé une concentration environnementale prévisible dans l'environnement qui indique le pourcentage de la dose d'application retombant sur la surface de l'eau à une distance déterminée de l'extrémité du pulvérisateur (Rivière, 2002). Ainsi, une fois ces bonnes pratiques sus-énumérées respectées, on pourra s'attendre à la durabilité agro-écologique des aménagements hydro-agricoles de la plaine inondable de la zone d'étude.

\section{Discussion}

L'échelle agro-écologique analyse la capacité du système de culture à combiner valorisation efficace du milieu et coût écologique minimum. Cette partie de la discussion est présentée selon les trois composantes de l'échelle de la durabilité.

\section{Composante « diversité »}

Les résultats des scores moyens obtenus de la composante diversité sont plus élevés sauf ceux obtenus sur l'indicateur diversité animale $(1,16)$. Cette faible valeur traduit l'inexistence des animaux domestiques sur les 
périmètres aménagés. Ce résultat ne concorde pas à celui de Ligan (2015) avec un score moyen de 10,7 sur 14 points, qui a étudié l'évaluation des exploitations agricoles dans la Commune de Gogounou. Cela peut s'expliquer par la prédominance des animaux domestiques (bovins, ovins, volailles et caprins) sur les exploitations agricoles au nord du Bénin. L'indicateur diversité des cultures annuelles et temporaires a eu un score de 11,2 à cause des différentes espèces végétales cultivées et de l'existence de la ressource en eau. Ces résultats sont confirmés par les travaux de Zannou (2017) réalisés dans la basse vallée de l'Ouémé au sud du Bénin. Concernant l'indicateur valorisation de l'espace et du patrimoine génétique, qui cherche non seulement à valoriser les espèces végétales ou animales rares ou menacées mais aussi àprévoir leur conservation, nos résultats ont fait cas de la présence de quelques espèces cités plus haut. On constate aisément que le score $(5,94)$ de cet indicateur est en dessus de celui de 0,29 obtenu par BekhoucheGuendouz (2011) dans les exploitations bovines laitières des bassins de la Mitidja et d'Annaba en Algérie. Ce faible score est dû au fait que les exploitants de la région de Mitidja ne valorisent pas les espèces végétales et animales d'origine ou de races régionales ou locales. Par contre, les résultats de cet auteur ont montré quelques espèces rares dans les exploitations des régions d'Annaba.

\section{Composante «Organisation de l'espace»}

Les scores 7,8 et 7,6 ont été obtenus respectivement des indicateurs assolement et zone de régulation écologique. Sur les périmètres, aucune surface de la principale culture annuelle en monoculture ne dépasse $35 \%$ de la surface assolable (4 points valeur de la méthode IDEA). L'assolement est caractérisé par la répartition des cultures maraîchères (tomate, légumes et gombo). La zone d'étude, située sur la plaine inondable, participe à une surface de régulation écologique. La présence des points d'eau et des zones humides facilitent le développement de la technique d'irrigation et de drainage. Les résultats de cet indicateur corroborent ceux de M'Hamdi et al. (2009) qui ont signalé l'importance de l'irrigation dans les exploitations laitières tunisiennes. Pour eux, les rendements des exploitations qui ne pratiquent pas l'irrigation sont aléatoires. La dimension des parcelles varie d'un périmètre à un autre. On y rencontre près de $10 \%$ des unités spatiales de dimension supérieure à 6 ha et une majorité moyennement inférieures ou égales à 8 ha. Le score de 4,8 accordé à cet indicateur confirme le même score obtenu par Ligan (2015) dans les exploitations agricoles de la Commune de Gogounou. Par ailleurs, la faune domestique étant quasiment inexistante sur les périmètres aménagés, aucune surface n'est destinée aux animaux. Or, la présence des animaux sur les périmètres aménages favorise une bonne gestion des surfaces fourragères. Ces résultats sont contraires à ceux de Lamothe (2007) qui a montré dans les 
exploitations agricoles françaises la disponibilité des surfaces agricoles ou des fourrages pour nourrir les lapins. Malheureusement, la majorité de ses lapins sont nourris de granulés complets provenant des industries, une situation que condamne la méthode IDEA.

\section{Composante «pratiques agricoles »}

Parmi les pratiques agricoles figure la fertilisation des sols par les engrais chimiques. Ces substances permettent pour la plupart d'accroître la croissance et participe à la formation des grains gros et riches en amidon. Pour nos résultats, $65 \%$ des enquêtés l'utilisent sur les périmètres aménagés. Les résultats de cet indicateur 2,78 sont différents de ceux trouvés par Rossier et al. (2001) qui ont montré dans leur étude sur le bilan écologique des exploitations agricoles, la pratique de la fertilisation organique au fumier plutôt qu'aux engrais chimiques. Dans la zone d'étude, on note une variété de pesticides ( 2,8 sur 10$)$ utilisés dans les périmètres. De nos jours, leur utilisation abusive a des répercussions sur le plan socio-environnemental. En effet, ces pratiques défavorables ne sont pas de nature à garantir à court, à moyen et à long terme la protection et la fertilité des sols. Ce qui est confirmé par le rapport (2016) du Programme d'Appui au Programme National d'Investissement de l'Agriculture au Sénégal. Dans ce rapport, il a été démontré l'utilisation des pesticides (avec un score de 3,1) dans les exploitations maraîchères dans le bassin arachidier du Sénégal.

\section{Conclusion}

La méthode IDEA est un outil d'aide pour la décision de la mise en œuvre de politiques publiques orientées vers le soutien de la durabilité des systèmes de production. L'étude permet de faire une auto-évaluation de la durabilité agro-écologique des aménagements hydro-agricoles dans les Communes de Dangbo et d'Adjohoun.

Cette évaluation a été possible grâce aux indicateurs des trois composantes de l'échelle de la durabilité. Toutefois, si bien les scores moyens des composantes « diversité » 23,88 et « organisation de l'espace »22,79 ont contribué à cette durabilité, les «pratiques agricoles»12,05 actuelles demeurent une composante sur laquelle des propositions d'exploitation doivent être faites pour une amélioration des aménagements hydro-agricoles vers plus de durabilité.

\section{References:}

1. Adam, K. S. \& Boko, M. (1993). Le Bénin, Edition Edicef, Paris, 96p.

2. Boko, M. (1988). Climats et communautés rurales du Bénin : Rythmes climatiques et rythmes de développement. Thèse de Doctorat d'Etat ès 
Lettres et Sciences Humaines. CRC, URA 909 du CNRS, Univ. de Bourgogne, Dijon, 2 volumes, 601p.

3. Bekhouche-Guendouz, N. (2011). "Evaluation de la Durabilité des Exploitations Bovines Laitières des Bassins de la Mitidja et d'Annaba ». Thèse de doctorat en Sciences Agronomiques, Ecole Nationale Supérieure d'Agronomie et des Industries Alimentaires (ENSAIA), Université de Nancy, 308 p.

4. Codjo, T. (2014). Aménagement hydro-agricoles pour la réduction de la vulnérabilité et l'adaptation de l'agriculture aux changements climatiques dans la Commune d'Adjohoun. Mémoire de Master IRD/FLASH/UAC, $96 \mathrm{p}$.

5. INSAE (2002). «Troisième Recensement Général de la Population et de l'Habitation », Cotonou, Bénin, tome II, 132 p.

6. Josien, E., Dobremez, L., \& Bidault, M-C. (2000). Multifonctionnalité et diagnostics d'exploitation dans le cadre des CTE: approche méthodologique et enseignements tirés des démarches adoptées dans quelques départements. Communication au séminaire «Premiers regards sur la multifonctionnalité de l'agriculture au travers de la mise en place des CTE », Clermont-Ferrand, 12-13 décembre.

7. Lalèyè, P. A. (1995). Ecologie comparée de deux espèces de chrysichthys, poissons siluriformes (Claroteidae) du complexe lagunaire lac Nokoué-lagune de Porto-Novo au Bénin. Université de Liège-Faculté des Sciences Agronomiques. Thèses de Doctorat, Belgique. $210 \mathrm{p}$.

8. Lamothe, F. L. (2007). Quelle est la durabilité de la production cunicole? Atouts et limites des conditions d'élevage actuelles. 12èmes Journées de la Recherche Cunicole, 27-28 novembre 2007, Le Mans, France, $12 \mathrm{p}$.

9. Landais, E. (1998). Agriculture durable : les fondements d'un nouveau contrat social ? Le courrier de l'environnement, $33 \mathrm{p}$.

10. Lègba, C.S. (2006). Pertinence de l'aménagement de la basse vallée de l'ouémé au Bénin. Mémoire de DESS. Institut International d'Ingénierie de l'eau et de l'environnement, 56p.

11. Ligan, T.F. (2015). Essai d'évaluation de la durabilité de l'agriculture : Application aux exploitations sous mécanisation agricole dans la Commune de Gogounou au nord Bénin. Thèse de doctorat en Géosciences de l'environnement et Aménagement de l'espace, Faculté des Sciences Humaines et Sociales, Université d'Abomey-Calavi, 283 p.

12. MAEP (2009). Annuaire statistique ; campagne agricole 2008-2009, pp 80-81. 
13. M'hamdi, N., Aloulou, R., Hedhly, M., \& Hamouda, M.B. (2009). «Evaluation de la durabilité des exploitations laitières tunisiennes par la méthode IDEA». Biotechnologie, Agronomie, Société et Environnement, 13 (2) 221-228.

14. Mw, H. \& Cherry, B. (2009). Death by multiple poisoning, glyphosate and Roundup. Science in Society, Vol. 42, N¹4, 9 p.

15. PAPSEN (2016). Analyse de la durabilité des exploitations maraîchères dans le bassin arachidier à travers la méthode IDEA. Rapport $n^{\circ} 26$ juin du Programme d'Appui au Programme National d'Investissement de l'Agriculture du Sénégal, $31 \mathrm{p}$.

16. Pierre, G., Madeline, P., Margetic, C., Croix, N., Bermond, M., \& Peltier, C. (2008). Durabilité, agricultures et territoires: quels questionnements pour les ruralistes d'universités de l'Ouest? Agricultures, durabilité, et territoires. Géocarrefour. Vol. 83, №3, 12p.

17. Pradel, M. \& Del'homme, B. (2005). Evaluation de la durabilité des exploitations viticoles dans le vignoble bordelais. Méthodes et résultats. In Actes du Congrès Oenometrics XII, 27-28 mai 2005, Macerata, Italie.

18. Renard, J.F., Cheik, L., \& Knips, V. (2004). L'élevage et l'intégration régionale en Afrique de l'ouest. Ministère des Affaires Etrangères, FAO-CIRAD, 37p.

19. Rivière, J.-L. (2002). Écotoxicologie et toxicologie des produits phytosanitaires. De l'obligation réglementaire à la protection des milieux, Ingénieries-EAT, $\mathrm{N}^{\circ}$ spécial 2001, p. 23-27.

20. Rossier, D. \& Gaillard, G. (2001). Bilan écologique de l'exploitation agricole. Méthode et application à 50 entreprises. Zurich-Reckenholz, Suisse : Station fédérale de recherches en agro-écologie et agriculture, $17 \mathrm{p}$.

21. SCDA (2016). Données statistiques de la campagne agricole. Rapport final, 78p.

22. Schwartz, D. (1995). Méthodes statistiques à l'usage des médecins et des biologistes. 4è édition (Editions médicales Flammarion), Paris, $314 \mathrm{p}$

23. Steinfeld, H., Gerber, P., Wassennaar, T., Castel, V., Rosales, M., \& De Haan, C. (2006). Livestock's long shadow-environnemental issues and options. Food and Agriculture Organization of the United Nations, Rome, Italie, $408 \mathrm{p}$.

24. Terriee, M., Gasselin, P., \& Le Blanc, J. (2010). Assessing the sustainability of activity systems to support agricultural households' projects. Methods and procedures for building sustainable farming systems, WS2.1, $11 \mathrm{p}$. 
25. Viliain, L., Bourdais, J-L., Boisset, K., Girardin, P., Guillaumin, A., Mouchet, C., Viaux P., \& Zahm (2000). La méthode IDEA Indicateurs de durabilité des exploitations agricoles- guide d'utilisation, Dijon, Educagri éditions, $99 \mathrm{p}$.

26. Vilain, L. (2003). La méthode IDEA-Indicateurs de durabilité des exploitations agricoles-guide d'utilisation. Deuxième édition. Educagri édition, $151 \mathrm{p}$.

27. Vilai, L. (2008). La méthode IDEA, indicateurs de durabilité des exploitations agricoles : guide d'utilisation. $3^{\text {ème }}$ éd. Educagri éditions, $184 \mathrm{p}$.

28. Vissin, W.E. (2013). Modélisation des eaux à des fins agricoles dans la basse vallée de l'Ouémé à Adjohoun (Bénin, Afrique de l'Ouest). In publication Annales FLASH, Vol 2, numéro 19, pp 96-107.

29. Zannou, D.V. (2017). Mise en valeur agricole et protection de l'environnement dans la basse de l'ouémé au sud du Bénin : une étude de durabilité écologique. Thèse de doctorat en Géosciences de l'environnement et Aménagement de l'espace, Faculté des Sciences Humaines et Sociales, Université d'Abomey-Calavi, 291p. 